\title{
Predicting Achromobacter Transport Pressured by Deposition of Carbon in Heterogeneous Sand Gravel Formation, Trans-Amadi Industrial Layout, Port Harcourt
}

\author{
Eluozo Solomon Ndubuisi ${ }^{1}$, Kenneth Miebaka Oba $^{2}$ \\ ${ }^{1}$ Department of Civil Engineering, Gregory University, Shawnee, Nigeria \\ ${ }^{2}$ Department of Civil Engineering, Rivers State University, Port Harcourt, Nigeria \\ E-mail address: \\ solondu2015@yahoo.com (E. S. Ndubuisi), Soloeluozo2013@hotmail.com (E. S. Ndubuisi)
}

\section{To cite this article:}

Eluozo Solomon Ndubuisi, Kenneth Miebaka Oba. Predicting Achromobacter Transport Pressured by Deposition of Carbon in Heterogeneous Sand Gravel Formation, Trans-Amadi Industrial Layout, Port Harcourt. International Journal of Biochemistry, Biophysics \& Molecular Biology. Vol. 2, No. 5, 2017, pp. 51-60. doi: 10.11648/j.ijbbmb.20170205.13

Received: October 2, 2016; Accepted: April 24, 2017; Published: October 28, 2017

\begin{abstract}
This paper expresses the predominant heterogeneous transport process of Achromobacter in the study area. These contaminant were monitored through mathematical modeling approach, the study were to predict the rate of concentration at different strata to unconfined bed, the expression from these derived model generated numerous theoretical values through simulation, the generated values through graphical representation expresses lots of variation in concentration at different formation, this condition were observed to reflect on the behaviour of the microbes in terms of transport influenced by predominant permeability coefficient, including deposition of micronutrient under predominant heterogeneous setting. The influences from this formation characteristics expresses the reflection of various rate of concentration observed in these figures, the deposition of Achromobacter through this developed model has definitely analyzed the level of transport in the study location, the study is imperative because experts will apply this developed tools in monitoring Achromobacter in soil and water environment.
\end{abstract}

Keywords: Predictive Model, Achromobacter, Heterogeneous and Formation

\section{Introduction}

It was predicted in 1995 that about 2.3 billion people (41\% of the world's population willed finitely experiences water stressed, Moreso it was predicted it will increase to 3.5 billion by 2025 ( $48 \%$ of the expected world population) [1]. This calls for demand implementation of other concept on way to maintained standard portable water [2]. They are utilized for other purpose such as waste water that consists of house hold waste water, industrial waste water, storm water is observed to seep to groundwater [11]. Most developed nation treat Wastewaters in some cities for industrial origin used for irrigate abroad multiplicity of crops and landscapes across the globe [5, 6, and 7]. However, efforts were made and most likely the best known approximation in 2001 was 20 million hectares of land which were irrigated with waste water partially diluted or undiluted $[8,9,10]$. There have been several outstanding reviews from other researchers on different aspects of waste water irrigation, including health impacts and risks $[12,13$, and 14], the ecological fate of organics $[9,10$, and 11], management of salts $[8,10,13]$ and public perceptions [3, 4, 7, and 8]. Inventories of waste water use in particular regions have been conducted [11, 12 , 13], but abroad over view of how many commonly found organic by-products in treated wastewater could affect agricultural productivity with persistent use in irrigation is lacking [13].

\section{Governing Equation}

$$
\alpha \tau \frac{d^{2} c}{d x^{2}}-\varphi u \frac{d c}{d x}+V_{o} \frac{d c}{d x}=0
$$

Nomenclature 
$\alpha \tau=$ Compressibility of mineral skeleton $\left[\mathrm{LT}^{2} \mathrm{M}^{-1}\right]$ $c=$ Agrobacterium $\left[\mathrm{ML}^{-3}\right]$

$\phi u=$ Porosity [-]

$V_{o}=$ Void Ratio [-]

$x=$ Distance $[\mathrm{L}]$

$$
\alpha \tau \frac{d^{2} c}{d x^{2}}-\left(\varphi u-V_{o}\right) \frac{d c}{d x}=0
$$

Let

$$
\begin{gathered}
C=\sum_{n=0}^{\infty} a_{n} x^{n} \\
C^{1}=\sum_{n=1}^{\infty} n a_{n} x^{n-1} \\
C^{11}=\sum_{n=2}^{\infty} n(n-1) a_{n} x^{n-2} \\
\alpha \tau n \sum_{n=2}^{\infty}(n-1) a_{n} x^{n-2}-\left(\varphi u-V_{o}\right) \sum_{n=1}^{\infty} n a_{n} x^{n-1}=0
\end{gathered}
$$

Replace $n$ in the $1^{\text {st }}$ term by $n+2$ and in the $2^{\text {nd }}$ term by $n+1$, so that we have;

$$
\alpha \tau \sum_{n=0}^{\infty}(n+2)(n+1) a_{n+2} x^{n}-\left(\varphi u-V_{o}\right) \sum_{n=0}^{\infty}(n+1) a_{n+1} x^{n}=0(4)
$$

i.e.

$$
\begin{gathered}
\alpha \tau(n+2)(n+1) a_{n+2}=\left(\varphi u-V_{o}\right)(n+1) a_{n+1} \\
a_{n+2}=\frac{\left(\varphi u-V_{o}\right)(n+1) a_{n+1}}{\alpha \tau(n+2)(n+1)} \\
a_{n+2}=\frac{\left(\varphi u-V_{o}\right) a_{n+1}}{\alpha \tau(n+2)}
\end{gathered}
$$

for

$$
n=0, a_{2}=\frac{\left(\varphi u-V_{o}\right) a_{1}}{2 \alpha \tau}
$$

for

$$
n=1, a_{3}=\frac{\left(\varphi u-V_{o}\right) a_{2}}{3 \alpha \tau}=\frac{\left(\varphi u-V_{o}\right)^{2} a_{1}}{2 \alpha \tau \bullet 3 \alpha \tau}
$$

for

$$
n=2 ; a_{4}=\frac{\left(\varphi u-V_{o}\right) a_{3}}{4 \alpha \tau}=\frac{\left(\varphi u-V_{o}\right)}{4 \alpha \tau} \bullet \frac{\left(\varphi u-V_{o}\right) a_{1}}{3 \alpha \tau \bullet 2 \alpha \tau}=\frac{\left(\varphi u-V_{o}\right)^{3} a_{1}}{4 \alpha \tau \bullet 3 \alpha \tau \bullet 2 \alpha \tau}
$$

for

$$
n=3 ; a_{5}=\frac{\left(\varphi u-V_{o}\right)}{5 \alpha \tau}=\frac{\left(\varphi u-V_{o}\right)^{4} a_{1}}{5 \alpha \tau \bullet 4 \alpha \tau \bullet 3 \alpha \tau \bullet 2 \alpha \tau}
$$

for

$$
n ; a_{n}-\frac{\left(\varphi u-V_{o}\right)^{n-1} a_{1}}{\alpha \tau^{n-1} n !}
$$

$$
C(x)=a_{0}+a_{1} x+a_{2} x^{2}+a_{3} x^{3}+a_{4} x^{4}+a_{5} x^{5}+\ldots \ldots . a_{n} x_{n}
$$

$$
\begin{gathered}
=a_{0}+a_{1} x+\frac{\left(\varphi u-V_{o}\right) a_{1} x^{2}}{2 ! \alpha \tau}+\frac{\left(\varphi u-V_{o}\right) a_{1} x^{3}}{3 ! \alpha \tau^{2}}+\frac{\left(\varphi u-V_{o}\right) x^{4}}{4 ! \alpha \tau^{3}}+\frac{\left(\varphi u-V_{o}\right)^{4}}{5 ! \alpha \tau^{4}} \\
C(x)=a_{0}+a_{1}\left[\frac{\left(\varphi u-V_{o}\right) x}{2 ! \alpha \tau}+\frac{\left(\varphi u-V_{o}\right)^{2} x^{3}}{3 ! \alpha \tau^{2}}+\frac{\left(\varphi u-V_{o}\right)^{3}}{4 ! \alpha \tau^{3}}+\frac{\left(\varphi u-V_{o}\right)^{4}}{5 ! \alpha \tau^{4}}\right] \\
C(x)=a_{0}+a_{1} e^{\frac{\left(\varphi u-V_{o}\right)}{\alpha \tau} x}
\end{gathered}
$$

Subject equation (16) to the following boundary condition

$$
\begin{gathered}
C(o)=0 \text { and } C^{1}(o)=L \\
C(x)=a_{0}+a_{1} \ell^{\frac{\left(\varphi u-V_{o}\right)}{\alpha \tau} x} \\
C(o)=a_{0}+a_{1}=0
\end{gathered}
$$

i.e.

$$
a_{0}+a_{1}=0
$$

$$
\begin{gathered}
C^{1}(x)=\frac{\left(\varphi u-V_{o}\right)}{2 ! \alpha \tau} a_{1} \ell^{\frac{\left(\varphi u-V_{o}\right)}{\alpha \tau} x} \\
C^{1}(o)=\frac{\left(\varphi u-V_{o}\right)}{2 ! \alpha \tau} a_{1}=L \\
a_{1}=\frac{L \alpha \tau}{\varphi u-V_{o}} \\
a_{1}=a_{0} \\
\Rightarrow a_{0}=\frac{-L \alpha \tau}{\varphi u-V_{o}}
\end{gathered}
$$

Hence, the particular solution of equation (16) is of the form:

$$
\begin{aligned}
C(x) & =-\frac{L \alpha \tau}{\varphi u-V_{o}}+\frac{L \alpha \tau}{\varphi u-V_{o}} \ell^{\frac{\left(\varphi u-V_{o}\right)}{\alpha \tau} x} \\
& \Rightarrow C(x)=\frac{L \alpha \tau}{\varphi u-V_{o}}\left[e^{\frac{\left(\varphi u-V_{t o}\right)}{\alpha \tau} x}-1\right]
\end{aligned}
$$


If

$$
x=V \bullet t
$$

Model at (20) can also be expressed in this form:

$$
\therefore C(x)=\frac{L \alpha \tau}{\varphi u-V_{o}}\left[\ell^{\frac{\left(\varphi u-V_{o}\right)}{\alpha \tau} V \bullet t}-1\right]
$$

Similarly, if $t=\frac{d}{v}$ from developed model at (21), it implies that the expression in (21) can be written as

$$
C(x)=\frac{L \alpha \tau}{\varphi u-V_{o}}\left[e^{\frac{\left(\varphi u-V_{o}\right)}{\alpha \tau} \frac{d}{V}}-1\right]
$$

If

$$
v=\frac{d}{t}
$$

$$
C(x)=\frac{L \alpha \tau}{\varphi-u-V_{o}}\left[e^{\frac{\left(\varphi u-V_{o}\right)}{\alpha \tau} \frac{d}{t}}-1\right]
$$

\section{Materials and Method}

Standard laboratory experiment where performed to monitor the concentration of chlorobium rate different formation, the soil deposition of the strata were collected in sequences base on the structural deposition at different locations, this samples were collected at different location, these samples generated variation at different depth producing different migration of Achromobacter concentration through pressure flow at lower end of the column, the experimental results are applied to be compared with the theoretical values to determined validation of the model.

\section{Results and Discussion}

Results and discussion are presented in tables including graphical representation of Achromobacter concentration.

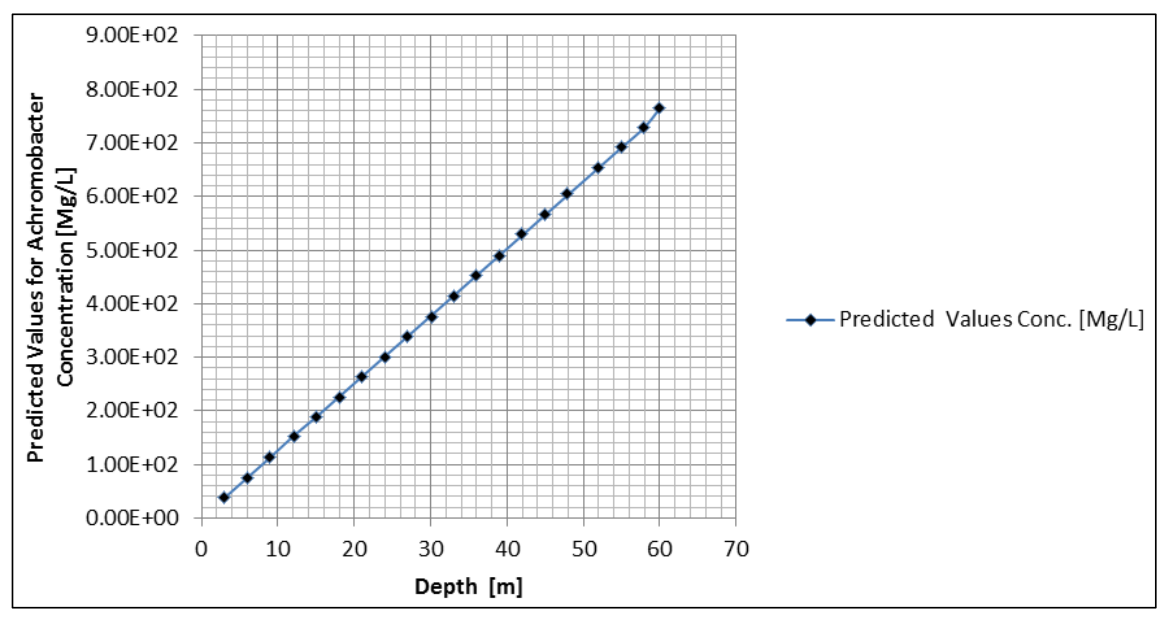

Figure 1. Concentration of Achromobacter at Different Depth.

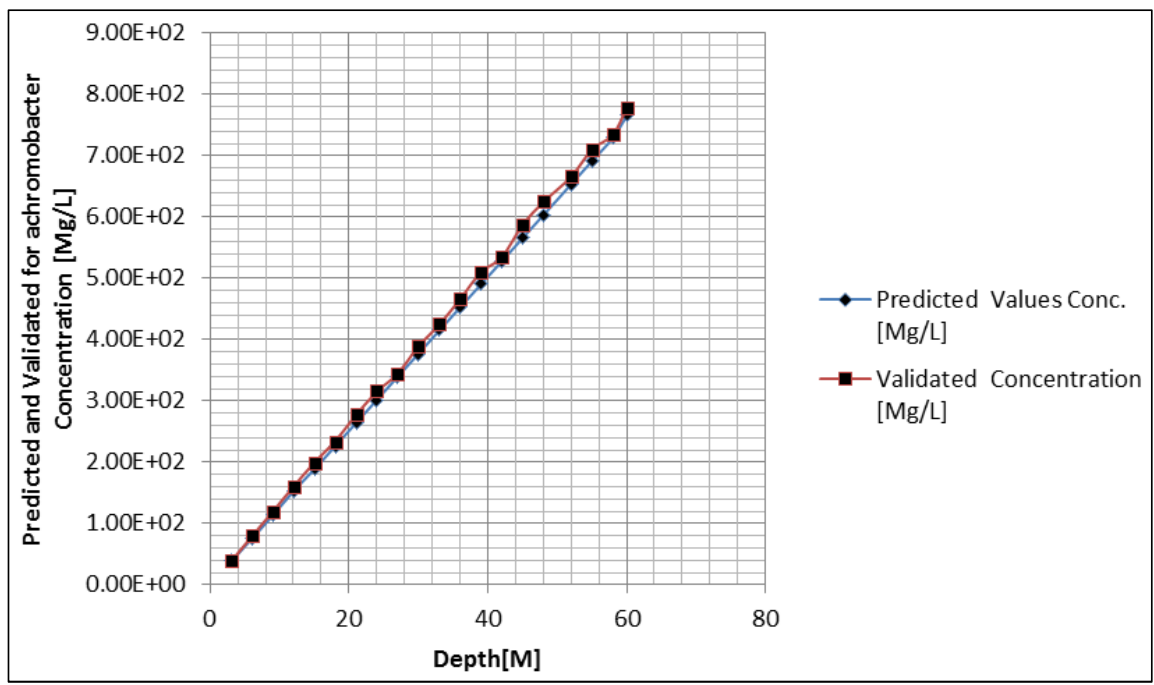

Figure 2. Predicted and Validate Concentration of Achromobacter at Different Depth 


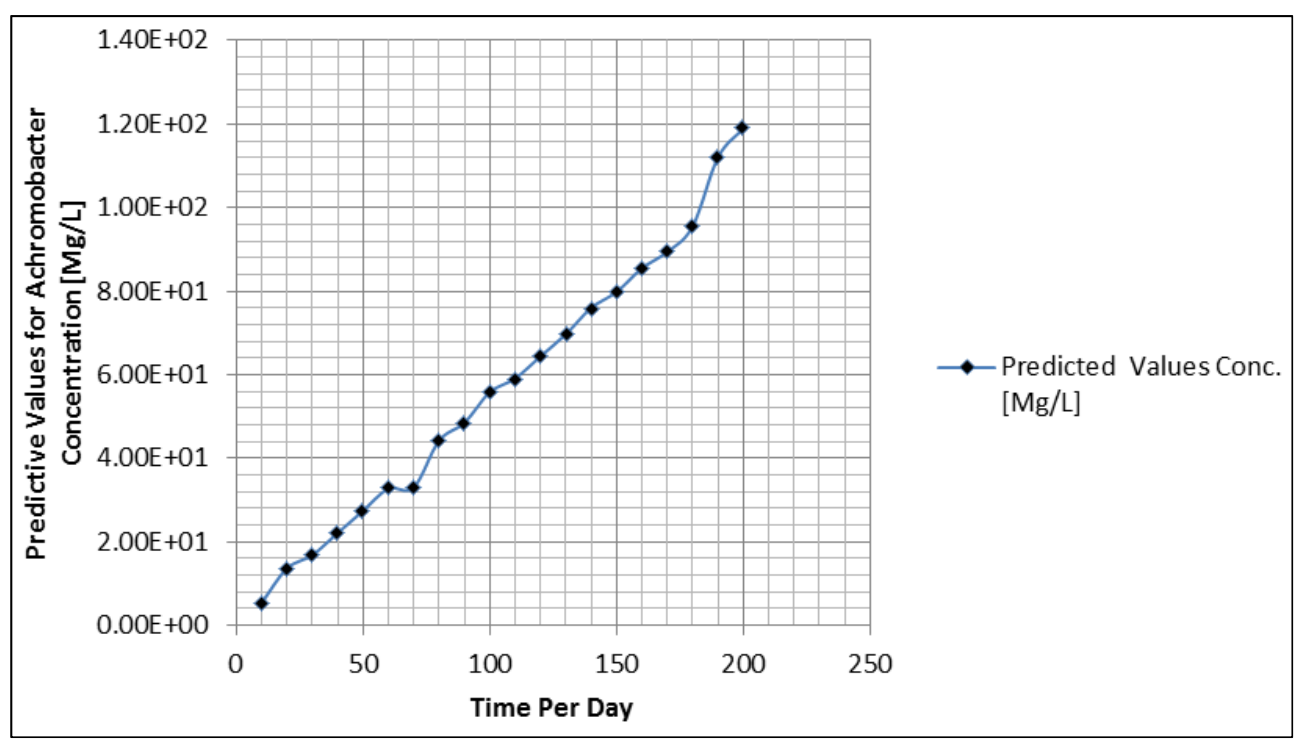

Figure 3. Concentration of Achromobacter at Different Depth.

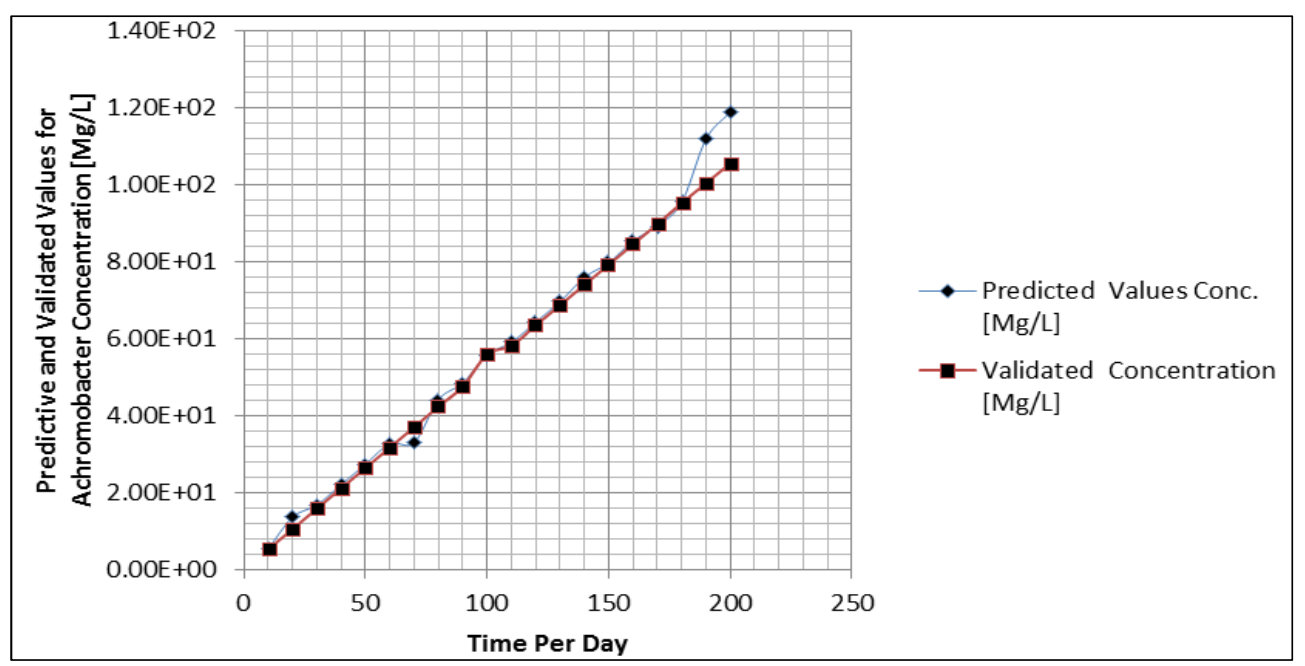

Figure 4. Predicted and Validate Concentration of Achromobacter at Different Depth.

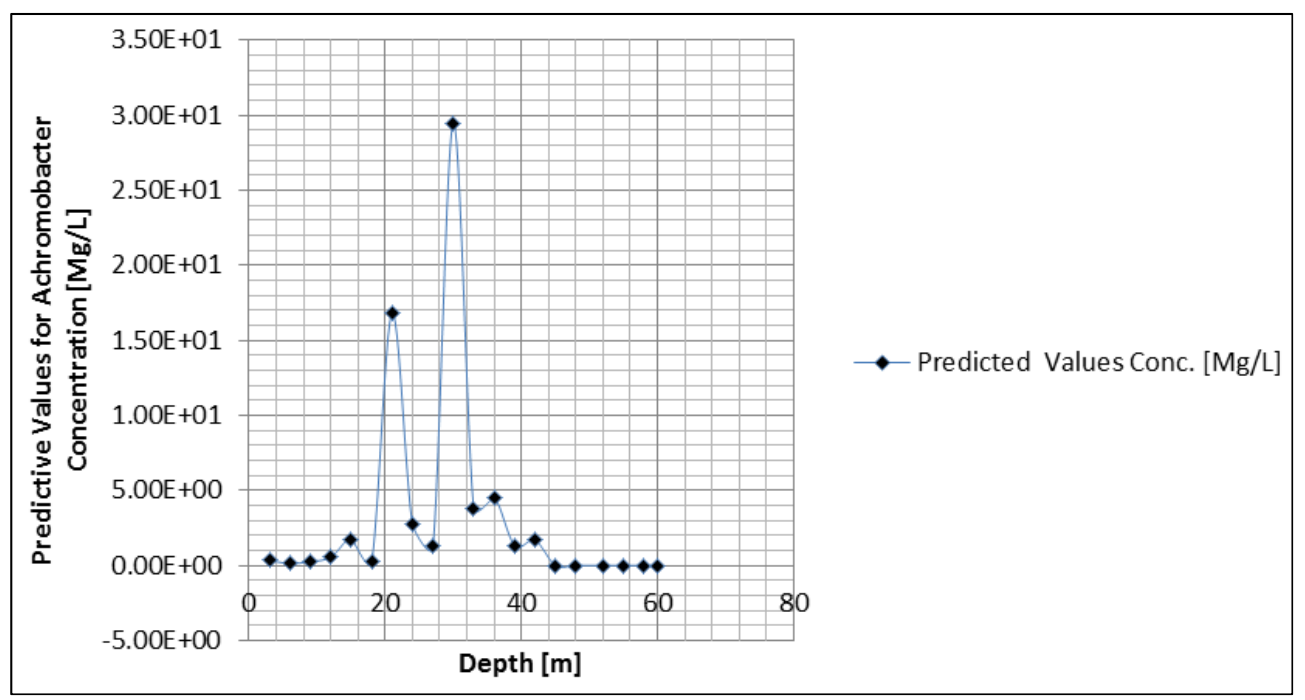

Figure 5. Concentration of Achromobacter at Different Depth. 

Carbon in Heterogeneous Sand Gravel Formation, Trans-Amadi Industrial Layout, Port Harcourt

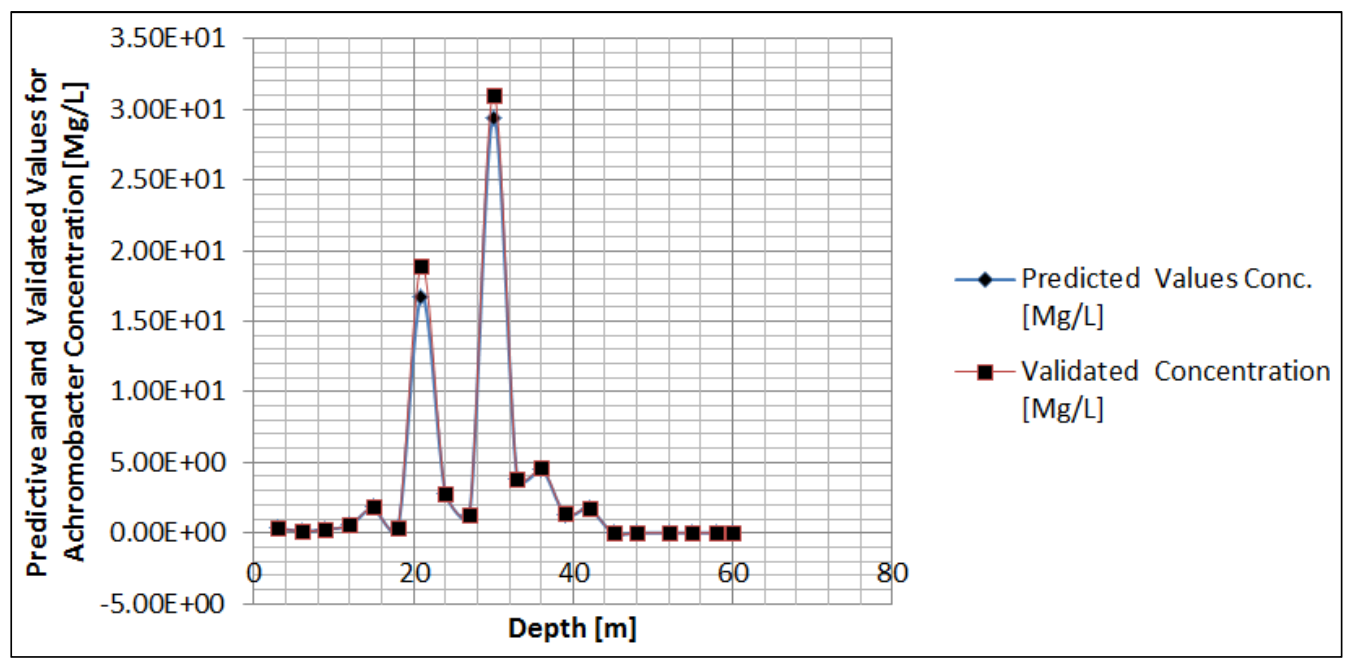

Figure 6. Predicted and Validate Concentration of Achromobacter at Different Depth.

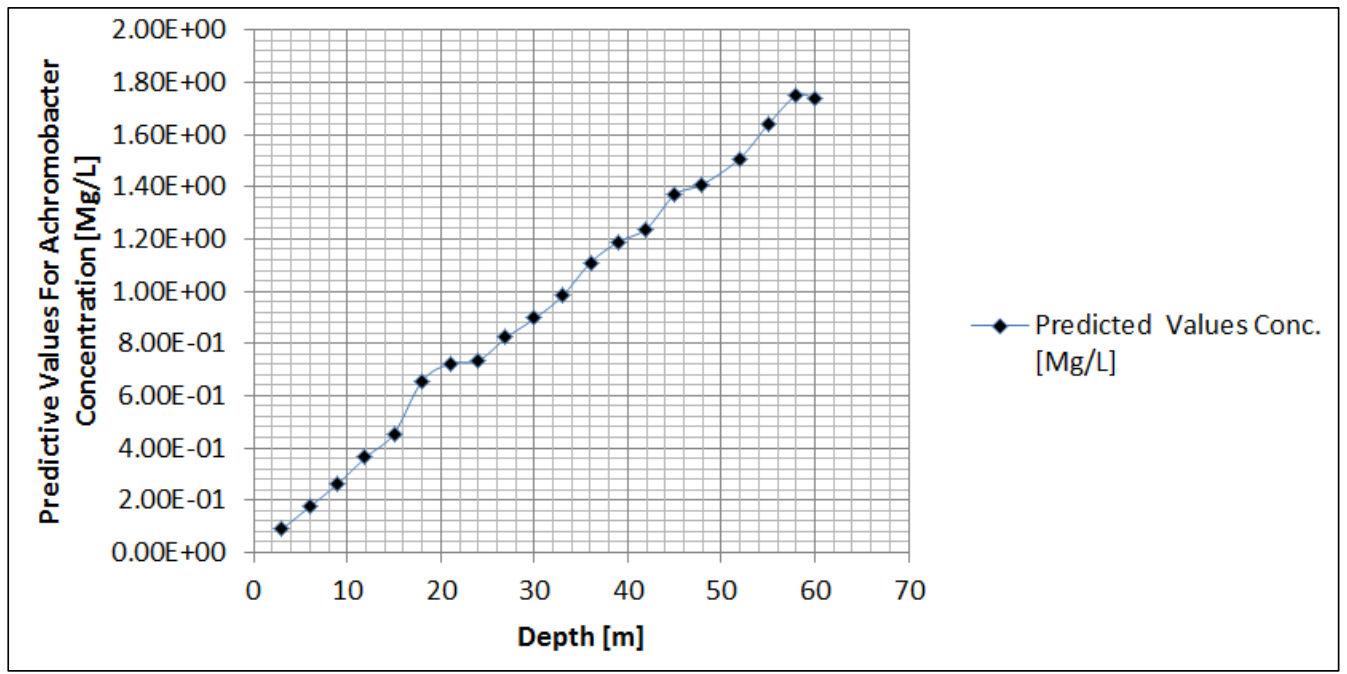

Figure 7. Concentration of Achromobacter at Different Depth.

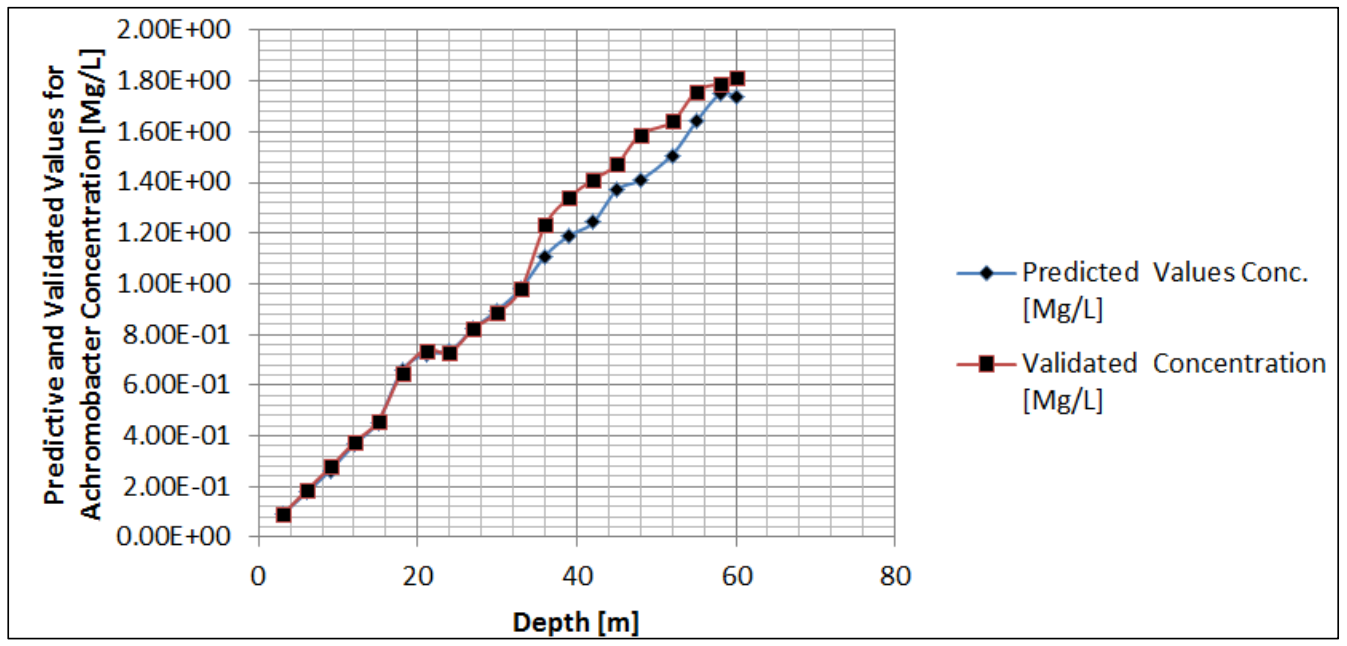

Figure 8. Predicted and Validate Concentration of Achromobacter at Different Depth. 


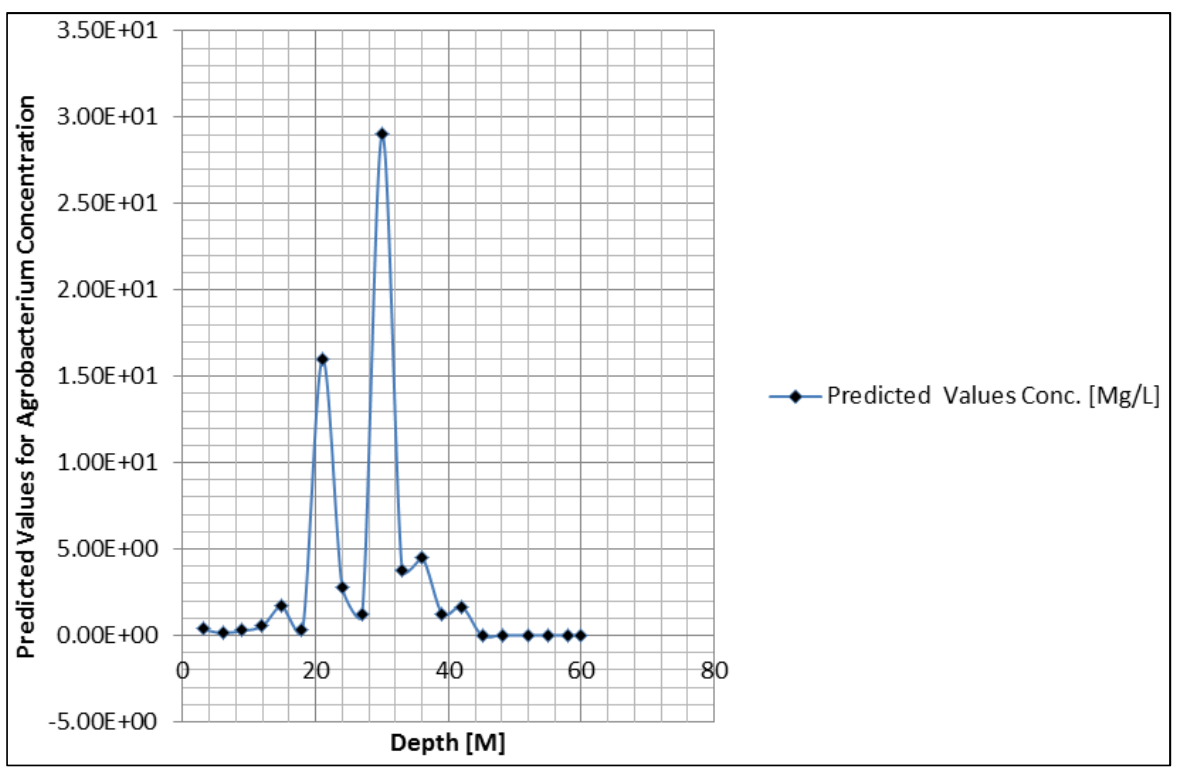

Figure 9. Concentration of Achromobacter at Different Depth.

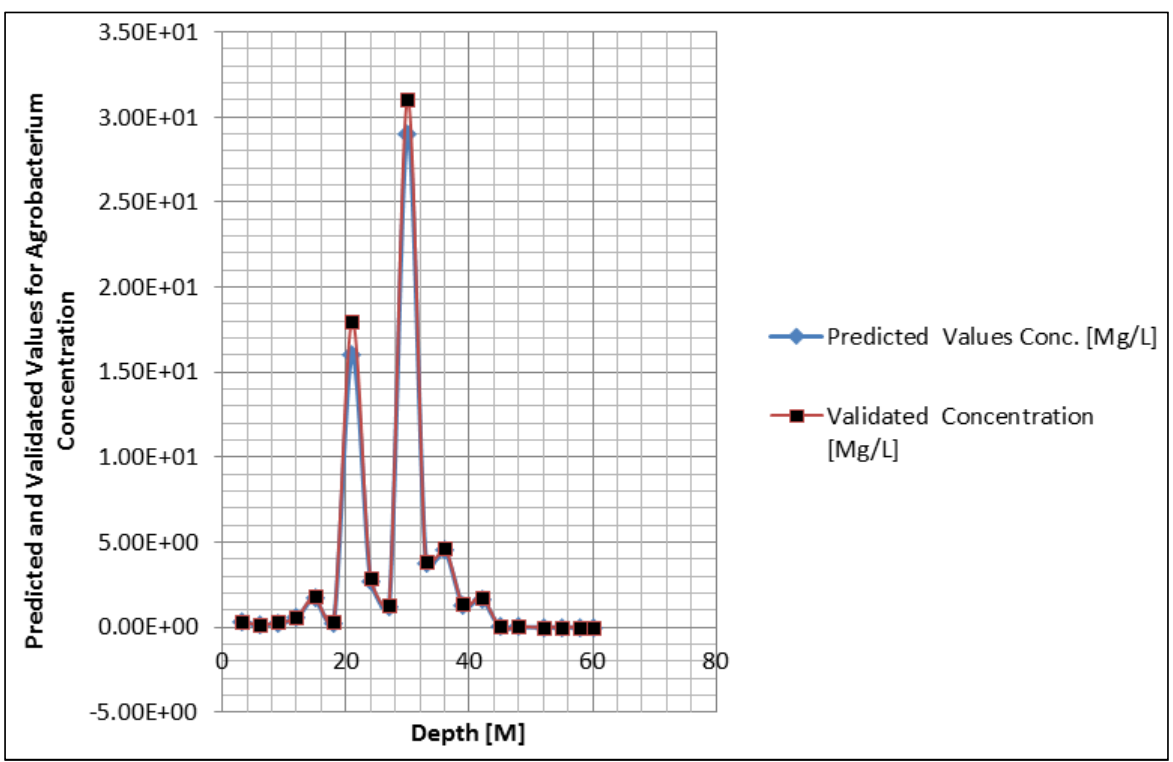

Figure 10. Predicted and Validate Concentration of Achromobacter at Different Depth.

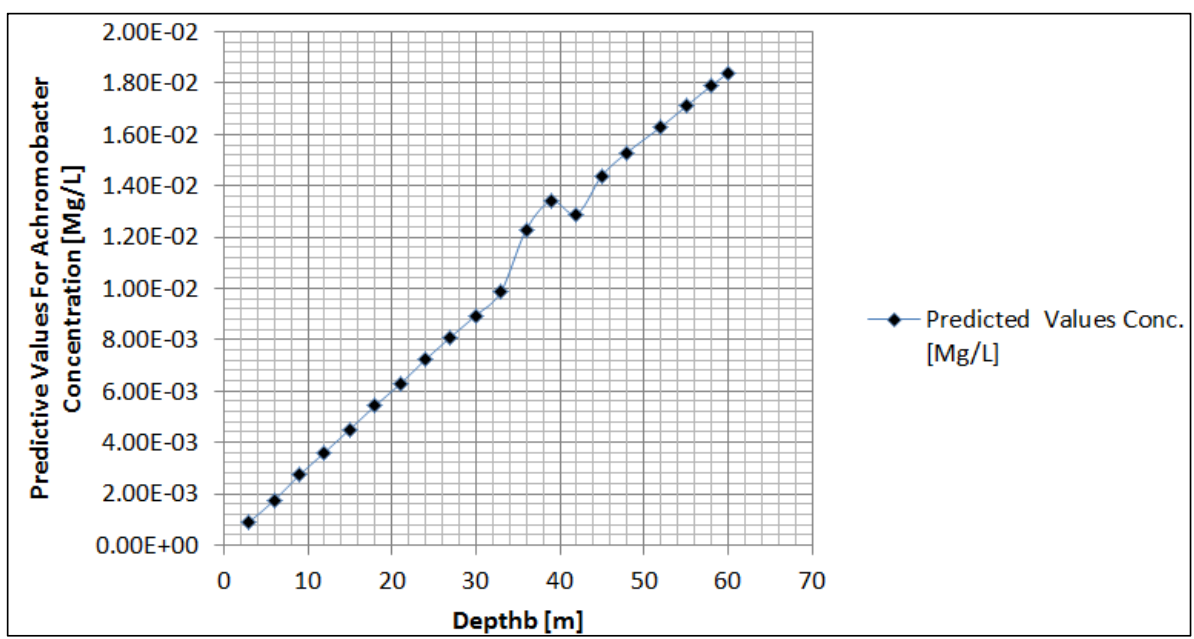

Figure 11. Concentration of Achromobacter at Different Depth. 


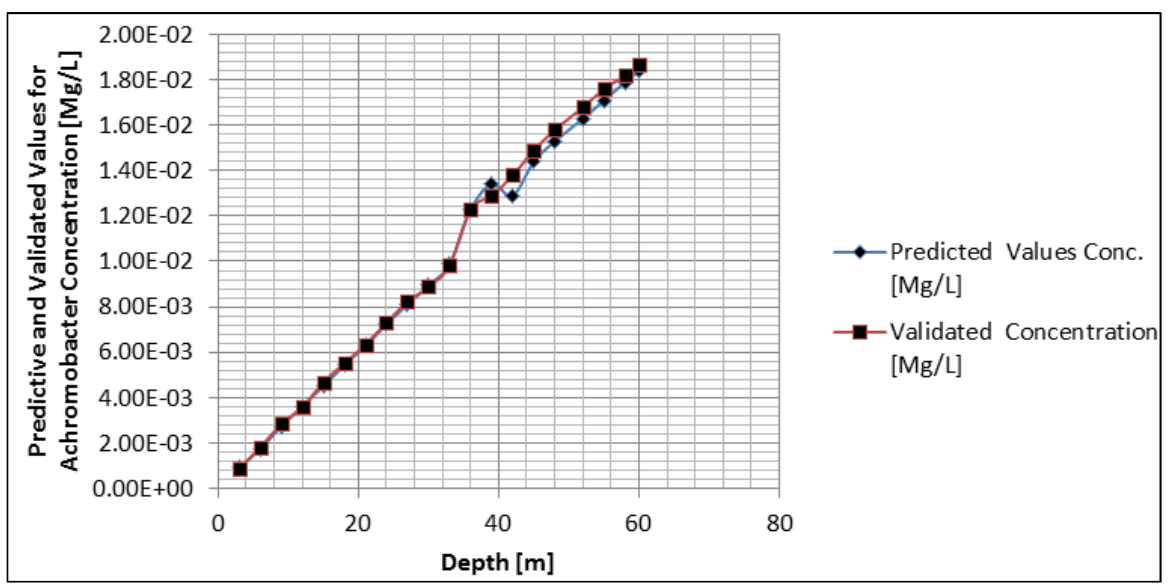

Figure 12. Predicted and Validate Concentration of Achromobacter at Different Depth.

Table 1. Concentration of Achromobacter at Different Depth.

\begin{tabular}{ll}
\hline Depth[M] & Predicted Values Conc. $[\mathbf{M g} / \mathbf{L}]$ \\
\hline 3 & $3.73 \mathrm{E}+01$ \\
6 & $7.56 \mathrm{E}+01$ \\
9 & $1.33 \mathrm{E}+02$ \\
12 & $1.55 \mathrm{E}+02$ \\
15 & $1.87 \mathrm{E}+02$ \\
18 & $2.24 \mathrm{E}+02$ \\
21 & $2.55 \mathrm{E}+02$ \\
24 & $3.11 \mathrm{E}+02$ \\
27 & $3.37 \mathrm{E}+02$ \\
30 & $3.74 \mathrm{E}+02$ \\
33 & $4.22 \mathrm{E}+02$ \\
36 & $4.58 \mathrm{E}+02$ \\
39 & $4.88 \mathrm{E}+02$ \\
42 & $5.22 \mathrm{E}+02$ \\
45 & $5.55 \mathrm{E}+02$ \\
48 & $6.13 \mathrm{E}+02$ \\
52 & $6.66 \mathrm{E}+02$ \\
55 & $6.86 \mathrm{E}+02$ \\
58 & $7.45 \mathrm{E}+02$ \\
60 & $7.68 \mathrm{E}+02$ \\
\hline
\end{tabular}

Table 2. Predicted and Validate Concentration of Achromobacter at Different Depth.

\begin{tabular}{lll}
\hline Depth[M] & Predicted Values Conc. $[\mathbf{M g} / \mathbf{L}]$ & $\begin{array}{l}\text { Validated } \\
\text { Concentration[Mg/L] }\end{array}$ \\
\hline 3 & $3.73 \mathrm{E}+01$ & 38.51 \\
6 & $7.56 \mathrm{E}+01$ & 79.34 \\
9 & $1.33 \mathrm{E}+02$ & 112.4 \\
12 & $1.55 \mathrm{E}+02$ & 164.4 \\
15 & $1.87 \mathrm{E}+02$ & 196.5 \\
18 & $2.24 \mathrm{E}+02$ & 231.6 \\
21 & $2.55 \mathrm{E}+02$ & 271.2 \\
24 & $3.11 \mathrm{E}+02$ & 312.5 \\
27 & $3.37 \mathrm{E}+02$ & 347.7 \\
30 & $3.74 \mathrm{E}+02$ & 388.3 \\
33 & $4.22 \mathrm{E}+02$ & 421.7 \\
36 & $4.58 \mathrm{E}+02$ & 463.2 \\
39 & $4.88 \mathrm{E}+02$ & 514.2 \\
42 & $5.22 \mathrm{E}+02$ & 538.7 \\
45 & $5.55 \mathrm{E}+02$ & 581.4 \\
48 & $6.13 \mathrm{E}+02$ & 627.7 \\
52 & $6.66 \mathrm{E}+02$ & 664.3 \\
55 & $6.86 \mathrm{E}+02$ & 715.3 \\
58 & $7.45 \mathrm{E}+02$ & 738.7 \\
60 & $7.68 \mathrm{E}+02$ & 774.8 \\
\hline
\end{tabular}

Table 3. Concentration of Achromobacter at Different Depth.

\begin{tabular}{ll}
\hline Time $[\mathbf{T}]$ & Predicted Values Conc. $[\mathbf{M g} / \mathbf{L}]$ \\
\hline 10 & $5.38 \mathrm{E}+00$ \\
20 & $1.36 \mathrm{E}+01$ \\
30 & $1.68 \mathrm{E}+01$ \\
40 & $2.21 \mathrm{E}+01$ \\
50 & $2.74 \mathrm{E}+01$ \\
60 & $3.27 \mathrm{E}+01$ \\
70 & $3.30 \mathrm{E}+01$ \\
80 & $4.42 \mathrm{E}+01$ \\
90 & $4.85 \mathrm{E}+01$ \\
100 & $5.58 \mathrm{E}+01$ \\
110 & $5.91 \mathrm{E}+01$ \\
120 & $6.44 \mathrm{E}+01$ \\
130 & $6.97 \mathrm{E}+01$ \\
140 & $7.60 \mathrm{E}+01$ \\
150 & $7.98 \mathrm{E}+01$ \\
160 & $8.55 \mathrm{E}+01$ \\
170 & $8.94 \mathrm{E}+01$ \\
180 & $9.55 \mathrm{E}+01$ \\
190 & $1.12 \mathrm{E}+02$ \\
200 & $1.19 \mathrm{E}+02$ \\
\hline
\end{tabular}

Table 4. Predicted and Validate Concentration of Achromobacter at Different Depth.

\begin{tabular}{lll}
\hline Time $[\mathbf{T}]$ & Predicted Values Conc. $[\mathbf{M g} / \mathbf{L}]$ & $\begin{array}{l}\text { Validated } \\
\text { Concentration }[\mathbf{M g} / \mathbf{L}]\end{array}$ \\
\hline 10 & $5.38 \mathrm{E}+00$ & 5.235 \\
20 & $1.36 \mathrm{E}+01$ & 10.528 \\
30 & $1.68 \mathrm{E}+01$ & 15.836 \\
40 & $2.21 \mathrm{E}+01$ & 21.128 \\
50 & $2.74 \mathrm{E}+01$ & 26.429 \\
60 & $3.27 \mathrm{E}+01$ & 31.728 \\
70 & $3.30 \mathrm{E}+01$ & 37.035 \\
80 & $4.42 \mathrm{E}+01$ & 42.41 \\
90 & $4.85 \mathrm{E}+01$ & 47.623 \\
100 & $5.58 \mathrm{E}+01$ & 56 \\
110 & $5.91 \mathrm{E}+01$ & 58.193 \\
120 & $6.44 \mathrm{E}+01$ & 63.57 \\
130 & $6.97 \mathrm{E}+01$ & 68.765 \\
140 & $7.60 \mathrm{E}+01$ & 74.058 \\
150 & $7.98 \mathrm{E}+01$ & 79.329 \\
160 & $8.55 \mathrm{E}+01$ & 84.594 \\
170 & $8.94 \mathrm{E}+01$ & 89.879 \\
180 & $9.55 \mathrm{E}+01$ & 95.35 \\
190 & $1.12 \mathrm{E}+02$ & 100.52 \\
200 & $1.19 \mathrm{E}+02$ & 105.698 \\
\hline
\end{tabular}


Table 5. Concentration of Achromobacter at Different Depth.

\begin{tabular}{ll}
\hline Time[T] & Predicted Values Conc.[Mg/L] \\
\hline 10 & $1.45 \mathrm{E}-01$ \\
20 & $1.39 \mathrm{E}+01$ \\
30 & $1.67 \mathrm{E}+01$ \\
40 & $1.39 \mathrm{E}+01$ \\
50 & $6.97 \mathrm{E}+00$ \\
60 & $1.47 \mathrm{E}+02$ \\
70 & $1.13 \mathrm{E}+01$ \\
80 & $3.14 \mathrm{E}+01$ \\
90 & $1.62 \mathrm{E}+02$ \\
100 & $1.57 \mathrm{E}+01$ \\
110 & $1.55 \mathrm{E}+01$ \\
120 & $7.16 \mathrm{E}-01$ \\
130 & $1.27 \mathrm{E}+00$ \\
140 & $1.38 \mathrm{E}+01$ \\
150 & $1.23 \mathrm{E}+00$ \\
160 & $2.36 \mathrm{E}-01$ \\
170 & $1.87 \mathrm{E}+01$ \\
180 & $9.91 \mathrm{E}+00$ \\
190 & $1.22 \mathrm{E}+01$ \\
200 & $1.23 \mathrm{E}+01$ \\
210 & $1.16 \mathrm{E}+02$ \\
220 & $1.30 \mathrm{E}+00$ \\
230 & $2.51 \mathrm{E}+00$ \\
\hline
\end{tabular}

Table 6. Predicted and Validate Concentration of Achromobacter at Different Depth.

\begin{tabular}{|c|c|c|}
\hline Time[T] & Predicted Values Conc.[Mg/L] & $\begin{array}{l}\text { Validated } \\
\text { Concentration }[\mathrm{Mg} / \mathrm{L}]\end{array}$ \\
\hline 10 & $1.45 \mathrm{E}-01$ & 0.471 \\
\hline 20 & $1.39 \mathrm{E}+01$ & 13.78 \\
\hline 30 & $1.67 \mathrm{E}+01$ & 16.53 \\
\hline 40 & $1.39 \mathrm{E}+01$ & 13.95 \\
\hline 50 & $6.97 \mathrm{E}+00$ & 7.56 \\
\hline 60 & $1.47 \mathrm{E}+02$ & 151.7 \\
\hline 70 & $1.13 \mathrm{E}+01$ & 14.8 \\
\hline 80 & $3.14 \mathrm{E}+01$ & 36.5 \\
\hline 90 & $1.62 \mathrm{E}+02$ & 172.8 \\
\hline 100 & $1.57 \mathrm{E}+01$ & 16.245 \\
\hline 110 & $1.55 \mathrm{E}+01$ & 16.79 \\
\hline 120 & $7.16 \mathrm{E}-01$ & 0.85 \\
\hline 130 & $1.27 \mathrm{E}+00$ & 1.46 \\
\hline 140 & $1.38 \mathrm{E}+01$ & 13.63 \\
\hline 150 & $1.23 \mathrm{E}+00$ & 1.18 \\
\hline 160 & $2.36 \mathrm{E}-01$ & 0.38 \\
\hline 170 & $1.87 \mathrm{E}+01$ & 18.55 \\
\hline 180 & $9.91 \mathrm{E}+00$ & 9.88 \\
\hline 190 & $1.22 \mathrm{E}+01$ & 10.23 \\
\hline 200 & $1.23 \mathrm{E}+01$ & 10.77 \\
\hline 210 & $1.16 \mathrm{E}+02$ & 122.18 \\
\hline 220 & $1.30 \mathrm{E}+00$ & 1.75 \\
\hline 230 & $2.51 \mathrm{E}+00$ & 2.78 \\
\hline 240 & $2.83 \mathrm{E}+00$ & 2.98 \\
\hline
\end{tabular}

\begin{tabular}{ll}
\hline Depth $[\mathbf{M}]$ & Predicted Values Conc. [Mg/L] \\
\hline 21 & $7.23 \mathrm{E}-01$ \\
24 & $7.34 \mathrm{E}-01$ \\
27 & $8.23 \mathrm{E}-01$ \\
30 & $8.95 \mathrm{E}-01$ \\
33 & $9.82 \mathrm{E}-01$ \\
36 & $1.11 \mathrm{E}+00$ \\
39 & $1.19 \mathrm{E}+00$ \\
42 & $1.24 \mathrm{E}+00$ \\
45 & $1.37 \mathrm{E}+00$ \\
48 & $1.41 \mathrm{E}+00$ \\
52 & $1.51 \mathrm{E}+00$ \\
55 & $1.64 \mathrm{E}+00$ \\
58 & $1.75 \mathrm{E}+00$ \\
60 & $1.74 \mathrm{E}+00$ \\
\hline
\end{tabular}

Table 8. Predicted and Validate Concentration of Achromobacter at Different Depth.

\begin{tabular}{lll}
\hline Depth $[\mathbf{M}]$ & $\begin{array}{l}\text { Predicted Values } \\
\text { Conc. }[\mathbf{M g} / \mathbf{L}]\end{array}$ & $\begin{array}{l}\text { Validated } \\
\text { Concentration }[\mathbf{M g} / \mathbf{L}]\end{array}$ \\
\hline 3 & $8.91 \mathrm{E}-02$ & $8.85 \mathrm{E}-02$ \\
6 & $1.75 \mathrm{E}-01$ & $1.84 \mathrm{E}-01$ \\
9 & $2.63 \mathrm{E}-01$ & $2.75 \mathrm{E}-01$ \\
12 & $3.65 \mathrm{E}-01$ & $3.74 \mathrm{E}-01$ \\
15 & $4.55 \mathrm{E}-01$ & $4.58 \mathrm{E}-01$ \\
18 & $6.56 \mathrm{E}-01$ & $6.48 \mathrm{E}-01$ \\
21 & $7.23 \mathrm{E}-01$ & $7.39 \mathrm{E}-01$ \\
24 & $7.34 \mathrm{E}-01$ & $7.28 \mathrm{E}-01$ \\
27 & $8.23 \mathrm{E}-01$ & $8.22 \mathrm{E}-01$ \\
30 & $8.95 \mathrm{E}-01$ & $8.84 \mathrm{E}-01$ \\
33 & $9.82 \mathrm{E}-01$ & $9.81 \mathrm{E}-01$ \\
36 & $1.11 \mathrm{E}+00$ & $1.23 \mathrm{E}+00$ \\
39 & $1.19 \mathrm{E}+00$ & $1.34 \mathrm{E}+00$ \\
42 & $1.24 \mathrm{E}+00$ & $1.41 \mathrm{E}+00$ \\
45 & $1.37 \mathrm{E}+00$ & $1.47 \mathrm{E}+00$ \\
48 & $1.41 \mathrm{E}+00$ & $1.59 \mathrm{E}+00$ \\
52 & $1.51 \mathrm{E}+00$ & $1.64 \mathrm{E}+00$ \\
55 & $1.64 \mathrm{E}+00$ & $1.76 \mathrm{E}+00$ \\
58 & $1.75 \mathrm{E}+00$ & $1.79 \mathrm{E}+00$ \\
60 & $1.74 \mathrm{E}+00$ & $1.81 \mathrm{E}+00$ \\
\hline
\end{tabular}

Table 9. Concentration of Achromobacter a Different Depth.

\begin{tabular}{ll}
\hline Depth[M] & Predicted Values Conc.[Mg/L] \\
\hline 3 & $3.46 \mathrm{E}-01$ \\
6 & $1.39 \mathrm{E}-01$ \\
9 & $2.69 \mathrm{E}-01$ \\
12 & $5.51 \mathrm{E}-01$ \\
15 & $1.77 \mathrm{E}+00$ \\
18 & $2.79 \mathrm{E}-01$ \\
21 & $1.68 \mathrm{E}+01$ \\
24 & $2.78 \mathrm{E}+00$ \\
27 & $1.27 \mathrm{E}+00$ \\
30 & $2.95 \mathrm{E}+01$ \\
33 & $3.79 \mathrm{E}+00$ \\
36 & $4.55 \mathrm{E}+00$ \\
39 & $1.34 \mathrm{E}+00$ \\
42 & $1.69 \mathrm{E}+00$ \\
45 & $1.29 \mathrm{E}-02$ \\
48 & $1.57 \mathrm{E}-02$ \\
52 & $7.33 \mathrm{E}-03$ \\
55 & $7.43 \mathrm{E}-03$ \\
58 & $8.34 \mathrm{E}-03$ \\
60 & $8.23 \mathrm{E}-04$ \\
\hline
\end{tabular}


Table 10. Predictedand Validate Concentration of Achromobacter at Different Depth

\begin{tabular}{lll}
\hline Depth $[\mathbf{M}]$ & $\begin{array}{l}\text { Predicted Values } \\
\text { Conc. }[\mathbf{M g} / \mathbf{L}]\end{array}$ & $\begin{array}{l}\text { Validated } \\
\text { Concentration }[\mathbf{M g} / \mathbf{L}]\end{array}$ \\
\hline 3 & $3.46 \mathrm{E}-01$ & 0.351 \\
6 & $1.39 \mathrm{E}-01$ & 0.141 \\
9 & $2.69 \mathrm{E}-01$ & 0.272 \\
12 & $5.51 \mathrm{E}-01$ & 0.561 \\
15 & $1.77 \mathrm{E}+00$ & 1.812 \\
18 & $2.79 \mathrm{E}-01$ & 0.282 \\
21 & $1.68 \mathrm{E}+01$ & 1900 \\
24 & $2.78 \mathrm{E}+00$ & 2.830 \\
27 & $1.27 \mathrm{E}+00$ & 1.311 \\
30 & $2.95 \mathrm{E}+01$ & 31.12 \\
33 & $3.79 \mathrm{E}+00$ & 3.842 \\
36 & $4.55 \mathrm{E}+00$ & 4.702 \\
39 & $1.34 \mathrm{E}+00$ & 1.393 \\
42 & $1.69 \mathrm{E}+00$ & 1.731 \\
45 & $1.29 \mathrm{E}-02$ & 0.019 \\
48 & $1.57 \mathrm{E}-02$ & 0.019 \\
52 & $7.33 \mathrm{E}-03$ & 0.00774 \\
55 & $7.43 \mathrm{E}-03$ & 0.00765 \\
58 & $8.34 \mathrm{E}-03$ & 0.00884 \\
60 & $8.23 \mathrm{E}-04$ & 0.000824 \\
\hline
\end{tabular}

Table 11. Concentration of Achromobacter at Different Depth.

\begin{tabular}{ll}
\hline Depth $[\mathbf{M}]$ & Predicted Values Conc. $[\mathbf{M g} / \mathbf{L}]$ \\
\hline 3 & $8.94 \mathrm{E}-04$ \\
6 & $1.74 \mathrm{E}-03$ \\
9 & $2.73 \mathrm{E}-03$ \\
12 & $3.61 \mathrm{E}-03$ \\
15 & $4.512 \mathrm{E}-03$ \\
18 & $5.43 \mathrm{E}-03$ \\
21 & $6.28 \mathrm{E}-03$ \\
24 & $7.23 \mathrm{E}-03$ \\
27 & $8.11 \mathrm{E}-03$ \\
30 & $8.95 \mathrm{E}-03$ \\
33 & $9.89 \mathrm{E}-03$ \\
36 & $1.23 \mathrm{E}-02$ \\
39 & $1.34 \mathrm{E}-02$ \\
42 & $1.29 \mathrm{E}-02$ \\
45 & $1.44 \mathrm{E}-02$ \\
48 & $1.53 \mathrm{E}-02$ \\
52 & $1.63 \mathrm{E}-02$ \\
55 & $1.71 \mathrm{E}-02$ \\
58 & $1.79 \mathrm{E}-02$ \\
60 & $1.84 \mathrm{E}-02$ \\
\hline
\end{tabular}

Table 12. Predicted and Validate Concentration of Achromobacter at Different Depth.

\begin{tabular}{lll}
\hline Depth $[\mathbf{M}]$ & $\begin{array}{l}\text { Predicted Values } \\
\text { Conc.[Mg/L] }\end{array}$ & $\begin{array}{l}\text { Validated Concentration } \\
\text { [Mg/L] }\end{array}$ \\
\hline 3 & $8.94 \mathrm{E}-04$ & $8.88 \mathrm{E}-04$ \\
6 & $1.74 \mathrm{E}-03$ & $1.81 \mathrm{E}-03$ \\
9 & $2.73 \mathrm{E}-03$ & $2.84 \mathrm{E}-03$ \\
12 & $3.61 \mathrm{E}-03$ & $3.58 \mathrm{E}-03$ \\
15 & $4.512 \mathrm{E}-03$ & $4.68 \mathrm{E}-03$ \\
18 & $5.43 \mathrm{E}-03$ & $5.52 \mathrm{E}-03$ \\
21 & $6.28 \mathrm{E}-03$ & $6.34 \mathrm{E}-03$ \\
24 & $7.23 \mathrm{E}-03$ & $7.31 \mathrm{E}-03$ \\
27 & $8.11 \mathrm{E}-03$ & $8.22 \mathrm{E}-03$ \\
30 & $8.95 \mathrm{E}-03$ & $8.87 \mathrm{E}-03$ \\
33 & $9.89 \mathrm{E}-03$ & $9.84 \mathrm{E}-03$ \\
36 & $1.23 \mathrm{E}-02$ & $1.23 \mathrm{E}-02$ \\
39 & $1.34 \mathrm{E}-02$ & $1.29 \mathrm{E}-02$ \\
42 & $1.29 \mathrm{E}-02$ & $1.38 \mathrm{E}-02$ \\
\hline
\end{tabular}

\begin{tabular}{lll}
\hline Depth $[\mathbf{M}]$ & $\begin{array}{l}\text { Predicted Values } \\
\text { Conc.[Mg/L] }\end{array}$ & $\begin{array}{l}\text { Validated Concentration } \\
{[\mathbf{M g} / \mathbf{L}]}\end{array}$ \\
\hline 45 & $1.44 \mathrm{E}-02$ & $1.49 \mathrm{E}-02$ \\
48 & $1.53 \mathrm{E}-02$ & $1.58 \mathrm{E}-02$ \\
52 & $1.63 \mathrm{E}-02$ & $1.68 \mathrm{E}-02$ \\
55 & $1.71 \mathrm{E}-02$ & $1.76 \mathrm{E}-02$ \\
58 & $1.79 \mathrm{E}-02$ & $1.82 \mathrm{E}-02$ \\
60 & $1.84 \mathrm{E}-02$ & $1.87 \mathrm{E}-02$ \\
\hline
\end{tabular}

Figure one and two presented express various transport rate of Achromobacter concentration in soil and water environment, the simulation values express its concentration with respect to time and depth thus in exponential level, figure three and four developed higher concentration due to the level of industrialization generating several type of waste situated in the study location, exponential phase was observed were the optimum values were recorded at period of two hundred days. Permeability coefficient were observed to deposit at higher rate, such predominant formation characteristics are reflected on the transport rates of the Achromobacter within these period to unconfined bed. Figure five and six expressed vacillation in some part of the study area, this was observed from the geological formation of the environment that deposited heterogeneous strata, therefore those area that experiences fluctuation are base on the deposition of microelements concentration, including porosity variation, such condition are reflected on the strata deposition in the transport system. Figure seven and eight were different compared to the previous one since homogeneous settings were observed with slight variation deposit. It experiences exponential migration, but the concentration were heterogeneous therefore the concentration maintained exponential phase. Figure nine and ten observed the predominant fluctuation base on the heterogeneity of the strata including the vacillation deposition of the micronutrient influences on the concentration. There reflection of these strata expresses the behaviour of the contaminant on their transport process, the concentration experienced fluctuation base on this influence from the strata. Figure eleven and twelve express linear migration base on some slight homogeneous setting found in some area of the study location, the deposition observed these influences but with oscillation in concentration at different depth of the formation, the concentration at these location cannot be compared to those other linear deposition in previous figures at the study area, the study is imperative because it has generated predicted values that shows the behaviour of Achromobacter in the study location.

\section{Conclusion}

The behaviour of Achromobacter in predominant heterogeneous formation has been investigated through modelling and simulation, these were achieved in these is conceptual framework that thoroughly represent the behaviour of the contaminant base on the effect from the geological depositions. This were monitored through the system in terms of it depositional phase in different strata, 
such condition were considered when the derived governing equation developed, some exponential phase were observed in the figures showing some slight homogeneous deposition in the study location, this also included the predominant formation characteristics such as permeability and deposition of micronutrients influences, these parameters pressure the Achromobacter transport process as it is reflected on its rate of concentration. The studies monitored the transport base on predominant heterogeneous influences from the formation that are observed in the environment. The vacillation of some figure expresses the pressure from the predominant heterogeneous strata in the area, the study is imperative because the refection of structural deposition of the formation has been observed through its rates of pressure on the migration process of Achromobacter in the study location.

\section{References}

[1] World Resources Institute (2000). Projected annual renewable water supply perpers on by river basin, 2025. Available at earth trends. wri. orgmaps_ spatial/ maps_detail_static.php?map_select $=265 \&$ theme $=4$ (verified7J anuary2010).

[2] Anderson J (2003). The environmental benefits of water recycling and reuse. Water Supply3: 1-10.

[3] Blumenthal U J. Peasey A (2002). Critical review of epidemiological evidence of the earth effects of waste water and excreta use in agriculture. London School of Hygiene and Tropical Medicine, London.

[4] Toze S (2006). Reuse of effluent water-benefits and risks. Agric. Water Manage. 80: 147-159.
[5] Müller K, Magesan G N, Bolan N. S (2007). A critical review of the influence of effluent irrigation on the fate of pesticides in soil. Agric. Ecosyst. Environ. 120: 93-116.

[6] Rengasamy P (2006). Soil salinity and sodicity. pp. 125-138. In Stevens (ed.) Growing crops with reclaimed wastewater. CSIRO Publ. Colling wood, Australia.

[7] Hartley TW (2003). Water reuse: Understanding public perception and participation. Water Environ. Res. Found. Alexandria, VA. pp. 115-126.

[8] PoM, Nancarrow B. E (2004). Consumer perceptions of the use of reclaimed water for horticultural irrigation. Aust. Res. Ctr. for Waterin Society, CSIRO, Perth, Australia.

[9] Angelakis A Thairs T, Lazarova V. (2001). Water reuse in EU countries: Necessity of establishing EU guidelines. Rep. EU201-26. EUREAU Water Reuse Group, Brussels.

[10] Bixio D, De Hyer B, Cikurel H, Muston M, Miska V, Joksimovic, Schäfer AI, Ravazzini A, Aharoni A, Savic D, Thoeye C (2005). Municipal wastewater reclamation: Where dowestand? Anoverview of treatment technology and management practice. Water Supply, 5: 77-85.

[11] Saqer A-S (1996). Environmental considerations for wastewater reuse in agriculture. Water Sci. Technol. 33 (1011): $345-353$.

[12] Radcliffe J C (2004). Water recycling in Australia. Aust. Acad. Technol. Sci. Eng. Parkville, Australia. 6: 823-840.

[13] Babatunde C. Akande, Patrick A. Ndakidemi, Olalekan Fatoki and James Odenda al 2010 The possible effect of the bioaccumulation of disinfectant by products on crops irrigated with treated wastewater African Journal of Biotechnology Vol. 9(9), pp. 1280-1287, 1 March, 2010. 